\title{
DIAGNÓSTICO DA HARMONIZAÇÃO DAS ESTRUTURAS DE CONTROLE INTERNO NA MARINHA DO BRASIL COM A ABORDAGEM COSO
}

\author{
Catia de Assis Silva das Chagas \\ Diretoria de Finanças da Marinha - Marinha do Brasil \\ Ilha das Cobras, $\mathrm{S} / \mathrm{N}^{0}-3^{\circ}$ andar - Centro - RJ - CEP 20091-000 \\ catiaachagas@gmail.com \\ Waldir Jorge Ladeira dos Santos \\ Universidade do Estado do Rio de Janeiro - UERJ \\ Rua São Francisco Xavier, 524 - $8^{\circ}$ andar - Bloco E - Maracanã - RJ - CEP 20550-900 \\ wcladeira@uol.com.br \\ José Francisco Moreira Pessanha \\ Universidade do Estado do Rio de Janeiro - UERJ \\ Rua São Francisco Xavier, 524 - 8º andar - Bloco E - Maracanã - RJ - CEP 20550-900 \\ professorjfmp@hotmail.com \\ Andréa Paula Osório Duque \\ Universidade do Estado do Rio de Janeiro - UERJ \\ Rua São Francisco Xavier, 524 - $8^{\circ}$ andar - Bloco E - Maracanã - RJ - CEP 20550-900 \\ andreapauladuque@gmail.com
}

\section{RESUMO}

O presente estudo buscou desenvolver um diagnóstico da harmonização das estruturas de controle interno na Marinha do Brasil (MB) com a abordagem preconizada pelo Committee of Sponsoring Organizations of the Theadway Commission (COSO). Dessa forma, a pesquisa foi definida como descritiva, com abordagem quantitativa. Realizou-se a coleta de dados por meio de um questionário estruturado e foram empregadas técnicas estatísticas multivariadas para análise de dados. Os resultados revelam que a maioria das Organizações Militares (OM) avaliadas apresentou boa harmonização com a abordagem COSO.

Palavra-chave: Controle Interno; Marinha do Brasil; COSO; Técnicas Estatísticas Multivariadas.

\section{ABSTRACT}

The present study sought to develop a diagnosis of the harmonization of internal control structures in the Brazilian Navy (MB) with the approach advocated by the Committee of Sponsoring Organizations of the Theadway Commission (COSO). Thus, the research was defined as descriptive, with quantitative approach. Data were collected through a structured questionnaire and multivariate statistical techniques were used for data analysis. The results show that most of the Military Organizations (OM) evaluated presented good harmonization with the COSO approach.

Keywords: KeywInternal control; Brazil's navy; COSO; Multivariate Statistical Techniques. 


\section{Como Citar:}

CHAGAS, Catia de Assis Silva das; SANTOS, Waldir Jorge Ladeira dos; PESSANHA, José Francisco Moreira; DUQUE, Andréa Paula Osório. Diagnóstico da Harmonização das Estruturas de Controle Interno na Marinha do Brasil com a Abordagem COSO. In: SIMPÓSIO DE PESQUISA OPERACIONAL E LOGÍSTICA DA MARINHA, 19., 2019, Rio de Janeiro, RJ. Anais [...]. Rio de Janeiro: Centro de Análises de Sistemas Navais, 2019.

\section{INTRODUÇÃO}

A atividade de controle interno funciona como um mecanismo de auxílio para a Administração Pública, a fim de conduzir ações de controle de forma econômica, eficiente e eficaz.

O controle interno nas organizações públicas iniciou com a publicação da Lei ${ }^{0}$ 4.320, de 17 de março de 1964. Essa lei estabelece normas para a elaboração e controle da execução orçamentária na Administração Pública, a fim de alcançar os resultados previstos (BRASIL, 1964). Brito (2009) afirma que a Lei $n^{\circ} 4.320 / 1964$ introduziu a expressão “controle interno".

Em seguida, a atividade de controle interno nos órgãos públicos foi reforçada e ampliada por meio da Constituição Federal (CF), de 1988 e da Lei Complementar $n^{\circ}$ 101, de 4 de maio de 2000, conhecida como Lei de Responsabilidade Fiscal (LRF).

Referindo-se à avaliação de controles internos, Crepaldi (2012) enfatiza que ela é um instrumento de incentivo que permite uma contínua melhoria nas atividades de controles internos.

É possível identificar, em estudos realizados, modelos sobre avaliação de controles internos, quais sejam: Committee of Sponsoring Organizations of the Theadwayc Commission (COSO), Control Objectives for Information and Related Technology (COBIT), Systems Auditabiliy and Control (SAC) e Statements on Auditing Standards (SAS) (GATTRINGER, 2016). Em face das melhorias existentes relacionadas ao controle interno, o modelo COSO apresenta os componentes "ambiente de controle”, "avaliação de riscos”, "atividades de controle", "informação e comunicação" e "atividades de monitoramento" (COSO, 2013). Esses componentes se configuram como o corpus deste estudo.

Com base no exposto, o estudo pretende responder as seguintes questões de pesquisa: As estruturas de controle interno na Marinha do Brasil (MB) estão harmonizadas com a abordagem COSO? Como avaliar a harmonização das estruturas de controle interno na Marinha do Brasil (MB) com a abordagem COSO? Nesta perspectiva, o objetivo do estudo consiste em desenvolver um diagnóstico da harmonização das estruturas de controle interno na Marinha do Brasil (MB) com a abordagem Committee of Sponsoring Organizations of the Theadwayc Commission (COSO).

A Marinha do Brasil (MB) foi escolhida, por ser uma instituição pública que contribui para o "preparo e a aplicação do Poder Naval em atividades relacionadas à ciência, tecnologia e inovação [...]”. Além disso, a MB é um dos órgãos públicos que planeja e controla a execução dos programas e ações previstos no orçamento da união (BRASIL, 2005).

O estudo sobre avaliação da harmonização das estruturas de controle interno no setor público se justifica, pois permite formular ações que visem a melhoria e o aperfeiçoamento contínuo das atividades de controle interno, bem como de sua estrutura. Além disso, pode contribuir para a eficiência e legitimidade das atividades de controle interno dos órgãos públicos (CONTI; CARVALHO, 2011), para o controle social do cidadão 
e das finanças públicas, uma vez que, ao avaliar o seu controle interno, as entidades públicas garantem a economicidade e a qualidade do gasto público (CADIN et al,, 2015).

A pesquisa está estruturada em cinco seções. Além desta introdução, a segunda seção apresenta o referencial teórico. A terceira seção descreve a metodologia. A quarta seção está dedicada à análise e discussão dos resultados. Por fim, na última seção, estão resumidas as principais conclusões do estudo.

\section{REFERENCIAL TEÓRICO}

Esta seção explora os pilares teóricos da pesquisa. Inicialmente é abordado o controle interno na Administração Pública e na sequência, é apresentado o modelo COSO. Ao final desta seção tem-se uma breve análise dos principais estudos sobre controle interno com a abordagem COSO.

\subsection{CONTROLE INTERNO NA ADMINISTRAÇÃO PÚBLICA}

O controle interno é uma das atividades realizada pela Administração Pública que possibilita o atingimento dos planos e das metas estabelecidas (CARDIN et., 2015).

O controle interno na Administração Pública, segundo Brandão (2015, p. 68), "surge da necessidade de corrigir rumos diante dos interesses da sociedade, e, atualmente, da necessidade de maior transparência dos gastos e da obrigação de uma utilização regular dos recursos públicos”.

Para Cardin et al. (2015), o controle interno é uma atividade exercida pelo gestor público que permite a economicidade e a qualidade do gasto público.

Os controles dos gastos nos órgãos públicos vêm evoluindo por meio “orçamentos, normas orientadoras e leis rígidas que prevê punições para uma má administração dos recursos públicos. Além de órgãos fiscalizadores em nível municipal, estadual e federal como a câmara municipal e o tribunal de contas” (SILVA; BONACIM, 2010, p. 153).

A Lei $n^{\circ} 4.320$, de 17 de março de 1964 é o normativo que introduziu a expressão “controle interno" (BRITO, 2009). Castro (2015, p. 387) acrescenta que essa lei “concentra toda a filosofia que justifica a existência de sistemas de controle ao determinar o controle sobre os atos administrativos, a fidelidade funcional dos agentes, o cumprimento dos limites financeiros e o alcance dos resultados”. Além disso, a Constituição Federal (CF), de 1988 e a Lei Complementar $\mathrm{n}^{\circ} 101$ (Lei de Responsabilidade Fiscal - LRF), de 4 de maio de 2000 fortaleceram o papel do controle interno na Administração Pública (BRASIL, 1988; BRASIL, 2000).

Chagas (2019) enfatiza que o controle interno, no setor público, vem sendo regularmente enriquecido por meio de outros normativos, quais sejam: Lei ${ }^{\circ} 8.666$, de 21 de junho de 1993 (Regula os procedimentos referentes a licitações e contratos) e a Resolução n ${ }^{0}$ 1.135, de 21 de novembro de 2008, do Conselho Federal de Contabilidade (CFC) (Estabelece aspectos sobre controle interno sob enfoque contábil).

\subsection{MODELO COSO}

Para avaliação do controle interno nas organizações, identifica-se o modelo COSO. Gattringer (2016) enfatiza que esse modelo é reconhecido no Brasil e no exterior como estrutura integrada de controle interno. Chagas (2019) destaca que o modelo COSO é utilizado no país, conforme Resolução ${ }^{\circ}$ 1.135, de 21 novembro de 2008, do CFC.

Para o COSO (2013, p. 7), o controle interno é "um processo conduzido pela estrutura de governança, pela administração e por outros profissionais da entidade, e desenvolvido para proporcionar segurança razoável com respeito à realização dos objetivos relacionados a operações divulgação e conformidade”. 
O modelo COSO, criado em 1985, nos Estados Unidos (EUA), é uma iniciativa do setor privado, composto por algumas entidades patrocinadoras, quais sejam: Associação Americana de Contabilidade (AAA); Instituto Americano de Contadores Públicos Certificados (AICPA); Executivos Financeiros Internacionais (FEI); Instituto de Contadores Gerenciais (IMA); e Instituto de Auditores Internos (IIA) (CHAGAS, 2019). A evolução histórica do modelo COSO é apresentada no Quadro 1, a seguir.

Quadro 1 - Evolução histórica do modelo COSO

\begin{tabular}{|c|l|}
\hline Modelos & \multicolumn{1}{|c|}{ Evolução histórica } \\
\hline COSO I & $\begin{array}{l}\text { Conhecido como Controle Interno - Estrutura Integrada. Objetivo: “melhorar a qualidade } \\
\text { dos relatórios financeiros, pela concentração de atenção na Governança Corporativa, nas } \\
\text { práticas éticas e nos Controles Internos” (TEIXEIRA, 2008, p. 81). }\end{array}$ \\
\hline \multirow{3}{*}{ COSO II } & $\begin{array}{l}\text { Conhecido como Gerenciamento de Riscos Corporativos - Estrutura Integrada. } \\
\text { Objetivo: Proporcionar uma estrutura de controle interno com maior visão na gestão de } \\
\text { riscos (CHAGAS, 2019). }\end{array}$ \\
\hline COSO III & $\begin{array}{l}\text { Versão atualizada da estrutura de controle interno composta por 17 princípios associados } \\
\text { aos cinco componentes (COSO, 2013). Objetivo: Proporcionar as organizações sistemas de } \\
\text { Controles internos eficazes que possibilitem o alcance de seus objetivos organizacionais } \\
\text { (CHAGAS, 2019). }\end{array}$ \\
\hline COSO - 2017 & $\begin{array}{l}\text { Atualização da estrutura de gerenciamento de riscos corporativos, publicado em 2004. } \\
\text { Objetivo: promover uma maior identificação dos riscos existentes a partir de ações } \\
\text { alinhadas aos objetivos e estratégias das instituições (COSO 2017). }\end{array}$ \\
\hline
\end{tabular}

Fonte: Os autores, 2019.

A seguir, o Quadro 2 apresenta os cinco componentes relacionados ao controle interno.

Quadro 2 - Componentes relacionados ao controle interno

\begin{tabular}{|c|l|}
\hline Componentes & \multicolumn{1}{c|}{ Abordagens } \\
\hline $\begin{array}{c}\text { Ambiente de } \\
\text { controle }\end{array}$ & $\begin{array}{c}\text { "Conjunto de normas, processos e estruturas que fornecem a base para a condução do } \\
\text { controle interno da organização" (CGU, 2017). }\end{array}$ \\
\hline Avaliação de riscos & $\begin{array}{l}\text { "Processo dinâmico e iterativo que visa a identificar, a analisar e a avaliar os riscos } \\
\text { relevantes que possam comprometer a integridade da Unidade Auditada e o alcance das } \\
\text { metas e dos objetivos institucionais” (CGU, 2017). }\end{array}$ \\
\hline $\begin{array}{c}\text { Atividades de } \\
\text { controle }\end{array}$ & $\begin{array}{l}\text { Ações são realizadas com objetivo de minimizar riscos existentes e atingir os objetivos } \\
\text { estabelecidos (COSO, 1994). }\end{array}$ \\
\hline $\begin{array}{c}\text { Informações e } \\
\text { comunicações }\end{array}$ & $\begin{array}{l}\text { As informações precisam ser identificadas e retransmitidas aos responsáveis e as pessoas } \\
\text { devem tomar conhecimento da importância desse componente no sistema de controle } \\
\text { interno da organização (COSO, 1994). }\end{array}$ \\
\hline $\begin{array}{c}\text { Atividades de } \\
\text { monitoramento }\end{array}$ & $\begin{array}{l}\text { Atividades realizadas ao longo do tempo, a fim de avaliar a qualidade do desempenho de } \\
\text { um determinado sistema de controle (COSO, 1994). }\end{array}$ \\
\hline
\end{tabular}

Fonte: Os autores, 2019.

Os componentes relacionados no Quadro 2 podem ser adotados em todas as organizações (COSO, 2013).

\subsection{PRINCIPAIS ESTUDOS ANTERIORES SOBRE CONTROLE INTERNO COM A ABORDAGEM COSO}

Araújo (2014) investigou o nível de alinhamento do sistema de controle interno na Universidade Federal da Paraíba (UFPA). O estudo identificou que os componentes monitoramento e avaliação de riscos possuem o menor índice de alinhamento do sistema de controle interno da Universidade. Foi apontado que o tempo de serviço, formação acadêmica e função exercida na Instituição são fatores que podem influenciar a percepção de alguns gestores. 
Cannavina (2014) estudou os sistemas de controles internos dos órgãos da Administração Pública Direta do Poder Executivo Federal (PEF). Os resultados apontam que há maior preservação do componente ambiente de controle e informação e comunicação. Dos componentes avaliados, o componente avaliação de riscos foi considerado o mais frágil. Observou-se que os órgãos avaliados não consideram os controles internos como uma ferramenta importante para atingir os objetivos previstos e melhorar o seu desempenho.

Menezes, Libonati e Neves (2015) analisaram o grau de similaridade dos elementos do sistema de controle interno da Universidade Federal de Pernambuco (UFPE). O estudo revelou que os componentes com coeficientes de similaridade com a abordagem COSO ERM (entre 40\% e 57\%) são "Ambiente de controle", "Fixação de objetivos" e "Identificação de eventos”. Já os componentes “Avaliação de riscos”, "Resposta a riscos”, "Procedimento controle", "Informação e comunicação" e "Monitoramento" apresentam coeficientes de dissimilaridade (entre 33\% e 38\%). O estudo constatou divergências conceituais entre os controles internos da Universidade e a estrutura de controle interno proposta pelo modelo COSO.

Gattringer (2016) avaliou os controles internos nos municípios catarinenses com o objetivo de "constatar se as variáveis relevantes de controles internos, relacionadas à eficiência operacional, estão substancialmente presentes”. O estudo revela que a escolaridade, o tempo de serviço no órgão e na função impactam a eficiência operacional do controle interno dos municípios catarinenses. Observou-se que os cinco componentes previstos no COSO são preservados pelos os municípios. Foi evidenciado que os sistemas de controles internos com base no COSO contribuem efetivamente para a eficiência operacional dos municípios catarinenses.

\section{METODOLOGIA}

\subsection{MÉTODO E POPULAÇÃO}

Para atingir o objetivo proposto, a pesquisa foi definida como descritiva. Para Malhotra (2012, p. 61), a pesquisa descritiva é “pré-planejada e estruturada”.

Quanto à abordagem do problema, o estudo é quantitativo. Segundo Malhotra (2012, p. 110), o estudo quantitativo "procura quantificar os dados e aplica alguma forma da análise estatística”. Diante disso, técnicas estatísticas multivariadas foram aplicadas, a fim de responder as questões de pesquisas propostas pelo estudo.

A instituição pública escolhida para a realização do estudo é a Marinha do Brasil (MB). A MB é uma instituição que contribui para "o preparo e a aplicação do Poder Naval em atividades relacionadas à ciência, tecnologia e inovação [...]”, bem como realiza o planejamento e a execução dos programas e metas previstas no orçamento da união (BRASIL, 2005).

A população da pesquisa é composta por 41 Organizações Militares (OM). Nas estruturas organizacionais dessas respectivas OM existem o Elemento de Controle Interno (ECI). Os ECI são os responsáveis técnicos para exercer as atividades de controle interno e monitorar os atos de gestão pública da OM.

As OM foram denominadas como OM1, OM2, OM3,...OM41, mantendo assim a transparência e veracidade dos dados coletados.

\subsection{COLETA E TRATAMENTO DOS DADOS}

Baseando-se nos estudos do TCU (2014) e de Gattringer (2016), a coleta de dados foi efetuada por meio de um questionário estruturado. A seção 1 contém questões específicas sobre controle interno com base na abordagem COSO, conforme indicado na Tabela 1. 
Seguindo as recomendações de Malhotra (2012), as perguntas estão ordenadas em escala Likert com cinco categorias de respostas, iniciando com 1 Discordo totalmente (DT) e terminando com 5 Concordo totalmente (CT). Já a seção 2 do questionário relaciona as questões referentes ao perfil dos respondentes.

Tabela 1 - Temas para avaliação da harmonização das estruturas de controle interno com a abordagem $\mathrm{COSO}$

\begin{tabular}{l|c|c}
\hline Temas & Componentes & Quantidade de perguntas \\
\hline Tema 1 & Ambiente de controle & 12 \\
\hline Tema 2 & Avaliação de riscos & 13 \\
\hline Tema 3 & Atividades de controle & 11 \\
\hline Tema 4 & Informação e comunicação & 09 \\
\hline Tema 5 & Atividades de Monitoramento & 05 \\
\hline Total & & $\mathbf{5 0}$ \\
\hline
\end{tabular}

Fonte: CHAGAS, 2019.

O questionário foi enviado por meio de mensagem interna contendo o link do Google Drive Formulários e disponibilizado no portal eletrônico do Centro de Controle Interno da Marinha (CCIMAR). O CCIMAR é a instituição da MB responsável pela execução das atividades de controle interno nas OM. A aplicação do questionário ocorreu no período de agosto a setembro de 2018.

Os dados foram analisados com o auxílio do Programa R. Sartório (2008) destaca que o R é um sistema desenvolvido para métodos interativos de análise de dados.

A partir do estudo de Neves, Pessanha e Santos (2013), o tratamento dos dados foi realizado por meio de técnicas estatísticas multivariadas (MINGOTI, 2017), conforme Figura 1.

Figura 1 - Aplicação de técnicas estatísticas multivariadas

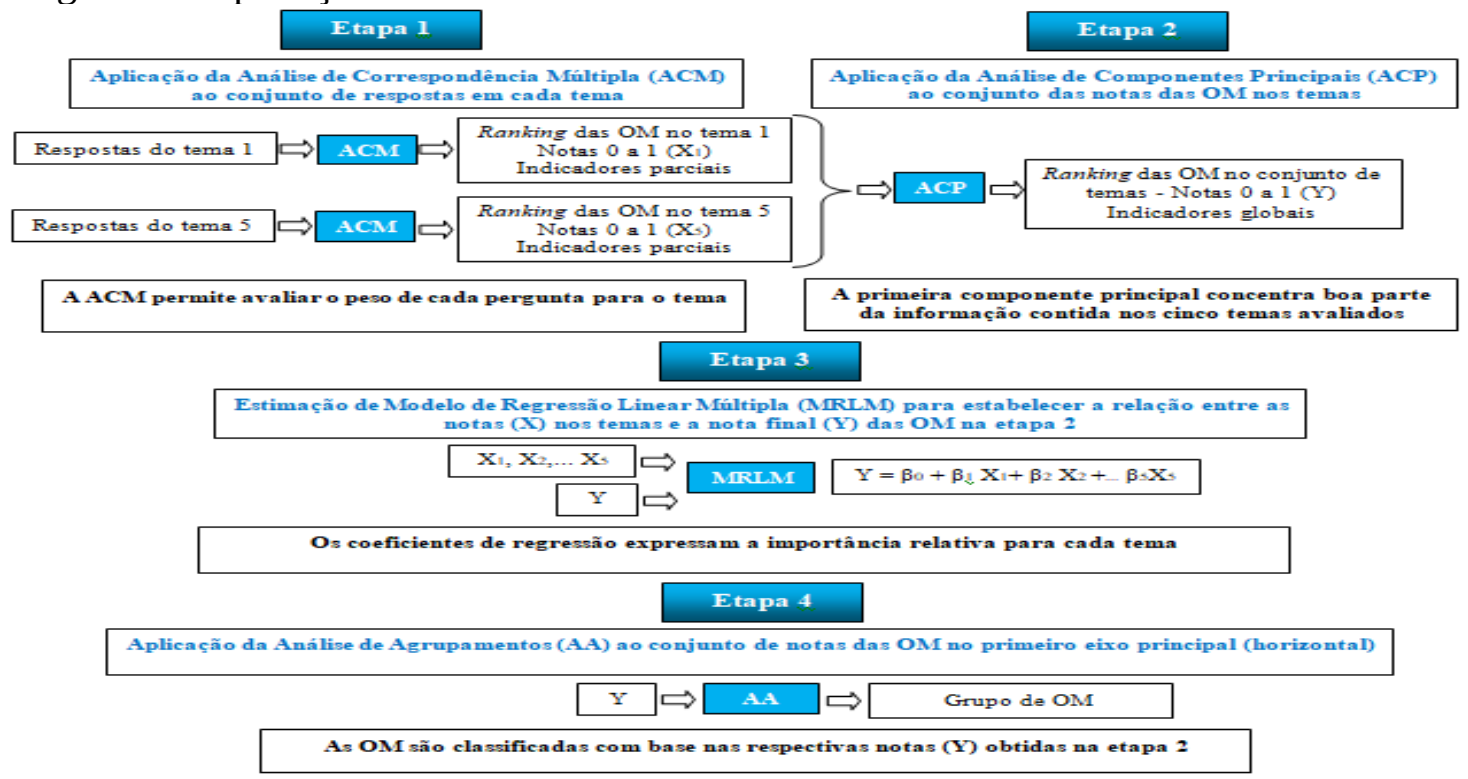

Fonte: CHAGAS, 2019.

A aplicação da ACM visa construir indicadores parciais da harmonização das Organizações Militares (OM) avaliadas com cada componente (tema) da abordagem COSO. A ACM é realizada individualmente com cada tema do questionário. Ao final desta etapa, as OM avaliadas recebem uma nota (indicadores parciais) que reflete a sua harmonização em cada tema (CHAGAS, 2019). 
Na sequência, na etapa 2, a aplicação da ACP ao conjunto dos cinco indicadores parciais visa obter um indicador global (Y) da harmonização com a abordagem COSO, capaz de sintetizar os diferentes componentes (temas) previstos na abordagem COSO (CHAGAS, 2019).

Na etapa 3, a importância relativa de cada componente (tema) no indicador global da harmonização é quantificada por meio de um MRLM. Segundo Chagas (2019), a importância relativa permite verificar a contribuição de cada componente para a harmonização das OM avaliadas com a abordagem COSO.

Por fim, a Etapa 4 (AA) consiste em segmentar as OM avaliadas em agrupamentos (clusters) semelhantes, bem como compará-los com a finalidade de identificar os respectivos pontos fracos e fortes referentes a harmonização com a abordagem COSO (CHAGAS, 2019).

\section{ANÁLISE E DISCUSSÃO DOS RESULTADOS}

Esta seção apresenta os resultados do presente estudo. Inicialmente, apresentam-se os resultados do perfil dos respondentes. Em seguida, apresenta-se a avaliação da harmonização das estruturas de controle interno na MB com a abordagem COSO.

\subsection{PERFIL DOS RESPONDENTES}

Quanto ao gênero dos respondentes da pesquisa, verifica-se que 75,6\% são do sexo masculino e $24,4 \% \%$ do sexo feminino.

Com relação à habilitação, 36,6\% dos respondentes possuem curso de mestrado e 24,4\% curso de especialização. Constata-se que 16 (32\%) dos respondentes têm formação acadêmica na área de administração e 15 (30\%) respondentes possuem curso na Escola Naval (EN). O curso de contabilidade está em terceiro lugar com 15 (14\%) respondentes.

Na questão quadro dos respondentes, observa-se que a maioria dos responsáveis pelo controle interno pertence ao Quadro de Intendência e Quadro Técnico (70\%). Assim, esta característica foi significativa para a avaliação da estrutura de controle interno nas OM. Chagas (2019) aponta que esses fatores contribuem para a avaliação das estruturas de controle interno das OM. A autora enfatiza que a pesquisa corrobora o estudo de Porto (2012) sobre avaliação da eficácia do sistema de controle interno no Comando da Aeronáutica. O estudo revela que a maioria dos Agentes de Controle Interno são da área de intendência (97\%).

Referindo-se aos postos ocupados nas atividades de controle interno, o estudo demonstra uma predominância de postos de nível superior $(58,5 \%)$ e de responsáveis pelo controle interno com mais de 15 anos de oficialato, alinhando à pesquisa de Porto (2012).

Na questão período de tempo na função pública, observou-se que metade da população (56,1\%) possui mais de 10 anos de função pública. Gattringer (2016) destaca que uma das variáveis relevantes para as atividades de controle interno é a o tempo de serviço dos gestores no setor público. Diante disso, Chagas (2019, p. 97) comenta que esse resultado pode "indicar aprimoramento de conhecimento e habilidades na execução e avaliação dos controles internos na MB”.

\subsection{AVALIAÇÃO DA HARMONIZAÇÃO DAS ESTRUTURAS DE CONTROLE INTERNO NA MB COM A ABORDAGEM COSO}

Conforme indicado na Figura 1, a análise dos dados provenientes da Seção 1 do questionário de pesquisa envolve quatro etapas, cada uma executada por uma determinada técnica estatística multivariada, cujos resultados são apresentados a seguir. 


\subsubsection{Aplicação da Análise de Correspondência Múltipla (ACM)}

As questões que compõem a Seção 1 do questionário de pesquisa estão organizadas em cinco blocos, cada um correspondendo a um tema da abordagem COSO. Assim, cada bloco de questões está associado com uma tabela, na qual cada linha corresponde à uma OM e cada coluna corresponde à uma questão do bloco de questões. Cada célula da tabela guarda a resposta de uma $\mathrm{OM}$ à uma questão, i.e., um valor entre 1 e 5 da escala de Likert de cinco pontos: Discordo totalmente (DT), Discordo parcialmente (DP), Não discordo nem concordo (NDNC), Concordo parcialmente (CP) e Concordo totalmente (CT) (CHAGAS, 2019). Inicialmente a ACM é aplicada separadamente em cada uma das cinco tabelas com o objetivo de produzir um mapa perceptivo que exibe as relações entre as questões em um mesmo bloco. Os mapas produzidos pela ACM para cada bloco são apresentados a seguir, começando na Figura 2 para o tema ambiente de controle e terminando na Figura 6 com o mapa perceptivo das questões sobre as atividades de monitoramento. Vale observar que no canto inferior esquerdo dos mapas perceptivos aparece a proporção da inércia total (informação) contida em cada mapa. Em todos os cinco temas avaliados os respectivos mapas concentram mais de $50 \%$ da inércia total presente nos dados, logo os mapas perceptivos fornecem um retrato razoável das associações entre as questões em um mesmo bloco de perguntas.

Figura 2 - Mapa perceptual obtido pela ACM no tema ambiente de controle

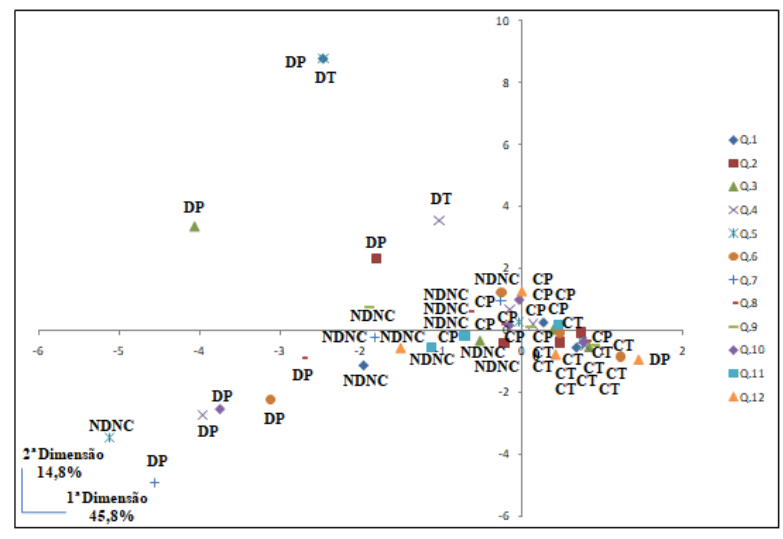

Fonte: CHAGAS, 2019.

Figura 4 - Mapa perceptual obtido pela ACM no tema atividades de controle

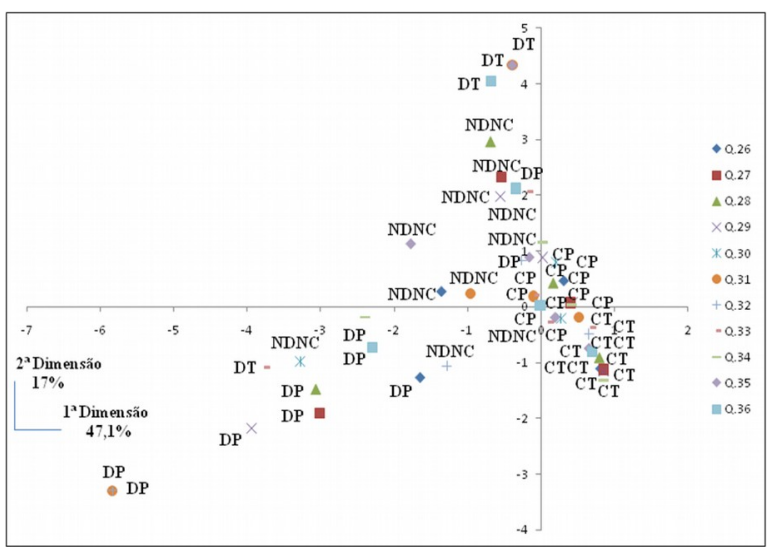

Fonte: CHAGAS, 2019.
Figura 3 - Mapa perceptual obtido pela ACM no tema avaliação de riscos

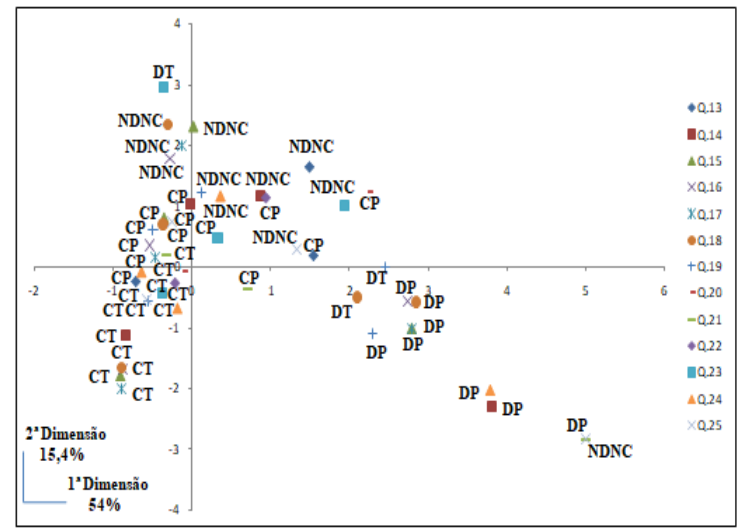

Fonte: CHAGAS, 2019.

Figura 5 - Mapa perceptual obtido pela ACM no tema informação e comunicação

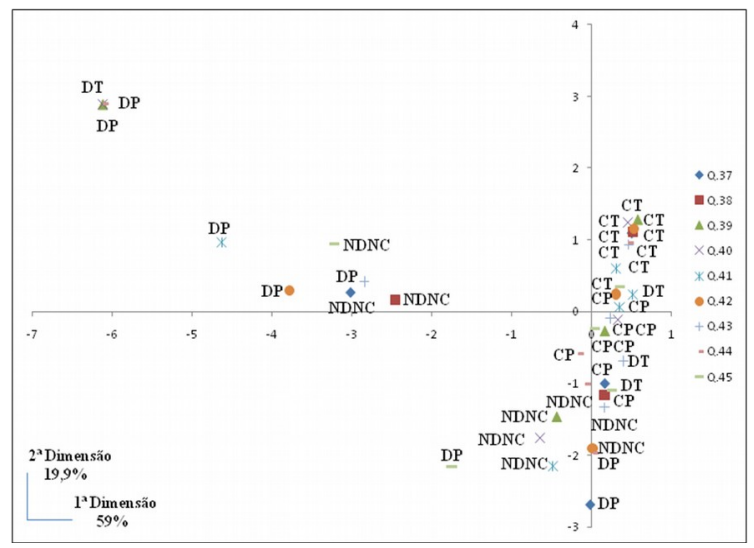

Fonte: CHAGAS, 2019. 
Figura 6 - Mapa perceptual obtido pela ACM no tema atividades de monitoramento

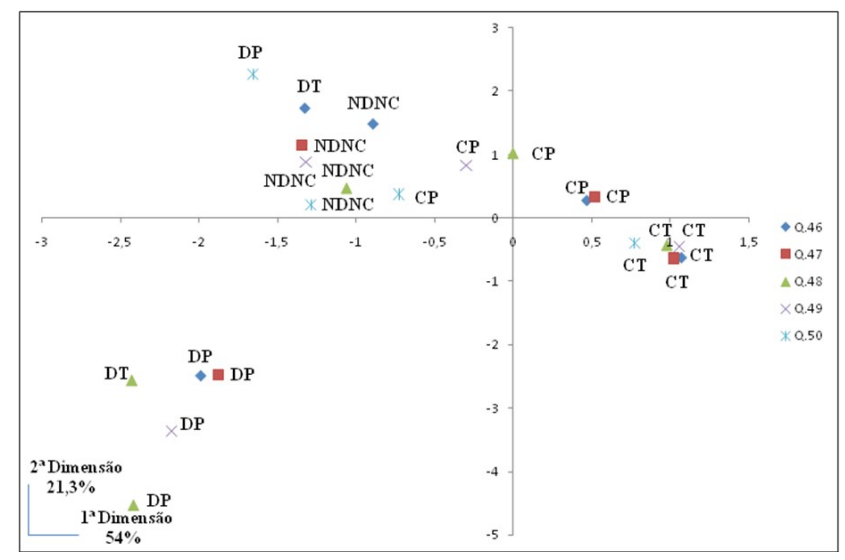

Fonte: CHAGAS, 2019.

No mapa ilustrado na Figura 2, as respostas DP estão concentradas no lado esquerdo do mapa perceptivo, enquanto as respostas CP e CT aparecem concentradas no lado direito do mapa. Tal ordenação sugere que a harmonização das OM avaliadas com o COSO tende a crescer no sentido da esquerda para a direita. Assim, o eixo horizontal do mapa perceptivo fornece um bom suporte para uma escala capaz de ordenar as OM quanto a harmonização com o COSO. As abscissas das OM avaliadas ao longo do eixo horizontal fornecem um indicador da harmonização das OM com o tema ambiente de controle. Interpretações semelhantes podem ser realizadas para os mapas ilustrados nas Figuras 4, 5 e 6, nas quais as respostas DT, DP, NDNC, CP e CT também apresentam uma ordenação ao longo do eixo horizontal no sentido da esquerda para a direita.

Já na Figura 3, a harmonização das OM no tema avaliação de riscos cresce no sentido da direita para a esquerda e mais uma vez o eixo horizontal fornece um bom suporte para a harmonização das OM com o tema avaliação de riscos.

As abscissas das OM em cada um dos cinco mapas fornecem os indicadores parciais que retratam a harmonização das OM com cada um dos temas do COSO. Os indicadores normalizados encontram-se na Tabela 2.

Tabela 2 - Indicadores parciais das OM nos cinco temas avaliados (continua)

\begin{tabular}{c|r|r|r|r|r}
\hline OM & $\begin{array}{c}\text { Ambiente de } \\
\text { controle }\end{array}$ & $\begin{array}{c}\text { Avaliação de } \\
\text { riscos }\end{array}$ & $\begin{array}{c}\text { Atividades de } \\
\text { controle }\end{array}$ & $\begin{array}{c}\text { Informação e } \\
\text { comunicação }\end{array}$ & $\begin{array}{c}\text { Atividades de } \\
\text { monitoramento }\end{array}$ \\
\hline OM1 & 0,54 & 0,65 & 0,64 & 0,56 & 0,34 \\
\hline OM2 & 0,65 & 0,56 & 0,61 & 0,54 & 0,59 \\
\hline OM3 & 0,67 & 0,69 & 0,67 & 0,56 & 0,55 \\
\hline OM4 & 0,66 & 0,69 & 0,68 & 0,62 & 0,78 \\
\hline OM5 & 0,33 & 0,14 & 0,45 & 0,45 & 0,33 \\
\hline OM6 & 0,38 & 0,48 & 0,47 & 0,44 & 0,26 \\
\hline OM7 & 0 & 0 & 0 & 0,07 & 0 \\
\hline OM8 & 0,45 & 0,50 & 0,32 & 0,50 & 0,17 \\
\hline OM9 & 0,65 & 0,64 & 0,19 & 0,58 & 0,50 \\
\hline OM10 & 0,22 & 0,40 & 0,30 & 0,43 & 0,72 \\
\hline OM11 & 0,67 & 0,63 & 0,66 & 0,61 & 0,49 \\
\hline OM12 & 0,55 & 0,51 & 0,41 & 0,63 & 0,72 \\
\hline OM13 & 0,54 & 0,65 & 0,60 & 0,55 & 0,42 \\
\hline OM14 & 0,71 & 0,65 & 0,57 & 0,56 & 0,14 \\
\hline OM15 & 0,35 & 0,05 & 0,43 & 0,50 & 0,75 \\
\hline OM16 & 0,61 & 0,68 & 0,56 & 0,59 & \\
\hline
\end{tabular}


Tabela 2 - Indicadores parciais das OM nos cinco temas avaliados (conclusão)

\begin{tabular}{c|r|r|r|r|r}
\hline OM & $\begin{array}{c}\text { Ambiente de } \\
\text { controle }\end{array}$ & $\begin{array}{c}\text { Avaliação de } \\
\text { riscos }\end{array}$ & $\begin{array}{c}\text { Atividades de } \\
\text { controle }\end{array}$ & $\begin{array}{c}\text { Informação e } \\
\text { comunicação }\end{array}$ & $\begin{array}{c}\text { Atividades de } \\
\text { monitoramento }\end{array}$ \\
\hline OM17 & 0,08 & 0,03 & 0,49 & 0,36 & 0,50 \\
\hline OM18 & 0,57 & 0,64 & 0,64 & 0,57 & 0,59 \\
\hline OM19 & 0,38 & 0,52 & 0,49 & 0,55 & 0,11 \\
\hline OM20 & 0,60 & 0,63 & 0,64 & 0,62 & 0,75 \\
\hline OM21 & 0,59 & 0,63 & 0,55 & 0,60 & 0,50 \\
\hline OM22 & 0,74 & 0,64 & 0,69 & 0,63 & 0,78 \\
\hline OM23 & 0,55 & 0,63 & 0,53 & 0,61 & 0,74 \\
\hline OM24 & 0,53 & 0,12 & 0 & 0 & 0,14 \\
\hline OM25 & 0,34 & 0,44 & 0,44 & 0,47 & 0,17 \\
\hline OM26 & 0,77 & 0,66 & 0,73 & 0,63 & 0,78 \\
\hline OM27 & 0,66 & 0,68 & 0,63 & 0,63 & 0,64 \\
\hline OM28 & 0,56 & 0,58 & 0,59 & 0,59 & 0,50 \\
\hline OM29 & 0,45 & 0,57 & 0,43 & 0,47 & 0,26 \\
\hline OM30 & 0,52 & 0,54 & 0,60 & 0,50 & 0,58 \\
\hline OM31 & 0,40 & 0,03 & 0,46 & 0,59 & 0,26 \\
\hline OM32 & 0,69 & 0,58 & 0,60 & 0,63 & 0,78 \\
\hline OM33 & 0,52 & 0,63 & 0,62 & 0,63 & 0,66 \\
\hline OM34 & 0,75 & 0,62 & 0,71 & 0,64 & 0,78 \\
\hline OM35 & 0,65 & 0,69 & 0,70 & 0,58 & 0,75 \\
\hline OM36 & 0,55 & 0,51 & 0,52 & 0,56 & 0,58 \\
\hline OM37 & 0 & 0,05 & 0 & 0 & 0,16 \\
\hline OM38 & 0,44 & 0,60 & 0,59 & 0,58 & 0,60 \\
\hline OM39 & 0,61 & 0,66 & 0,64 & 0,57 & 0,72 \\
\hline OM40 & 0,75 & 0,68 & 0,71 & 0,61 & 0,74 \\
\hline OM41 & 0,61 & 0,62 & 0,65 & 0,59 & 0,74 \\
\hline
\end{tabular}

Fonte: CHAGAS, 2019.

Adicionalmente, na ACM é possível verificar as contribuições de cada questão nos temas avaliados para índice que mensura a harmonização das $\mathrm{OM}$ avaliadas com a abordagem COSO (CARVALHO, 2008), conforme Figura 7.

Figura 7 - Contribuições das questões no tema ambiente de controle

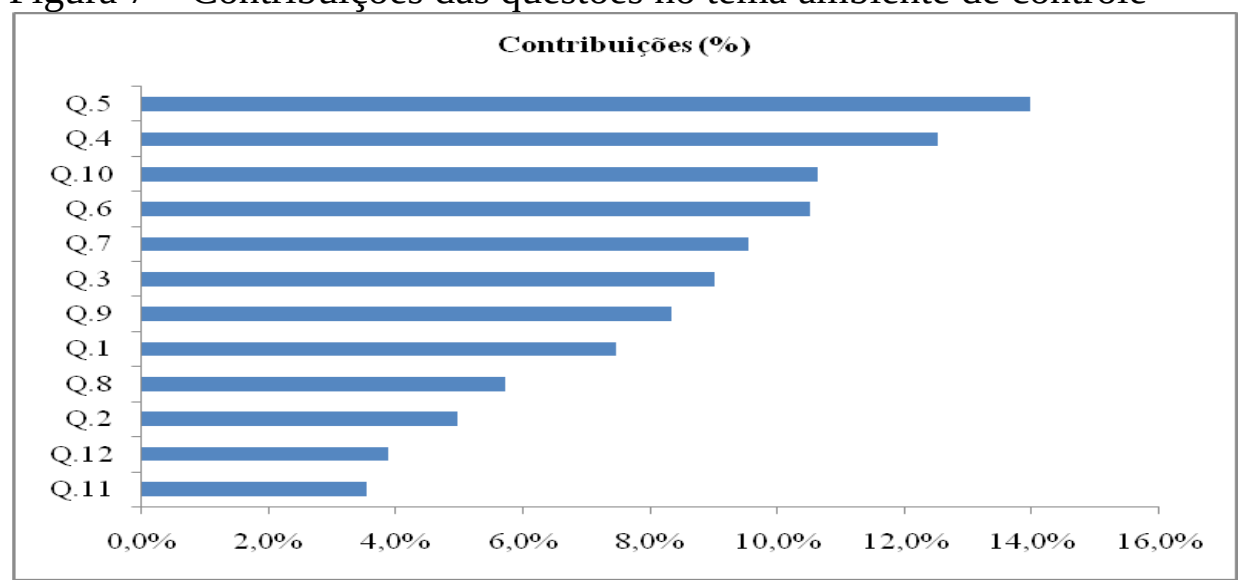

Fonte: CHAGAS, 2019.

Por exemplo, conforme ilustrado na Figura 7, as principais contribuições no tema ambiente de controle são a padronização dos procedimentos e instruções operacionais (Q.5), a existência do código de ética ou conduta (Q.4) e a adoção um programa de capacitação, treinamento e conscientização (Q.10). 
A mensuração e classificação dos riscos (Q.18), os níveis de riscos, de informações e de conformidade (Q.16), a realização de diagnósticos de riscos estratégicos e a probabilidade dos mesmos ocorrerem (Q.15) e a avaliação contínua de riscos internos e externos (Q.17) são as questões que mais contribuíram para a harmonização no tema avaliação de riscos. Já com relação ao tema atividades de controle é possível observar a representatividade de atividades de controle abrangentes e relacionadas com os objetivos de controle da organização (Q.29), de funcionamento de atividades de controle conforme o plano de longo prazo (Q.27), de adoção de atividades de controle com uma apropriação de custo ao nível de benefícios que possam derivar de sua aplicação (Q.28) e de execução de controle por meio de sistemas de informática e TI (Q.32).

No tema informação e comunicação, a comunicação das informações presentes em todos os níveis hierárquicos (Q.41), a comunicação adequada e eficiente referente ao controle interno (Q. 42) e a divulgação interna da informação (Q.40) se apresentam como as mais representativas. Por fim, a adequabilidade e efetividade do sistema de controle interno (Q.47) e o seu monitoramento constante, a fim de avaliar sua validade e qualidade ao longo do tempo (Q.46) foram os fatores que mais contribuíram no tema atividades de monitoramento.

\subsubsection{Resultados da Análise de Componentes Principais (ACP)}

Na sequência, por meio da aplicação da ACP, os indicadores parciais obtidos pela ACM (Tabela 2) foram sintetizados em um indicador global de harmonização das OM com o COSO.

A Figura 8 apresenta as notas das OM por meio do indicador global, classificandoas segundo a harmonização das estruturas de controle interno com a abordagem COSO.

Figura 8 - Ranking das OM segundo o indicador global

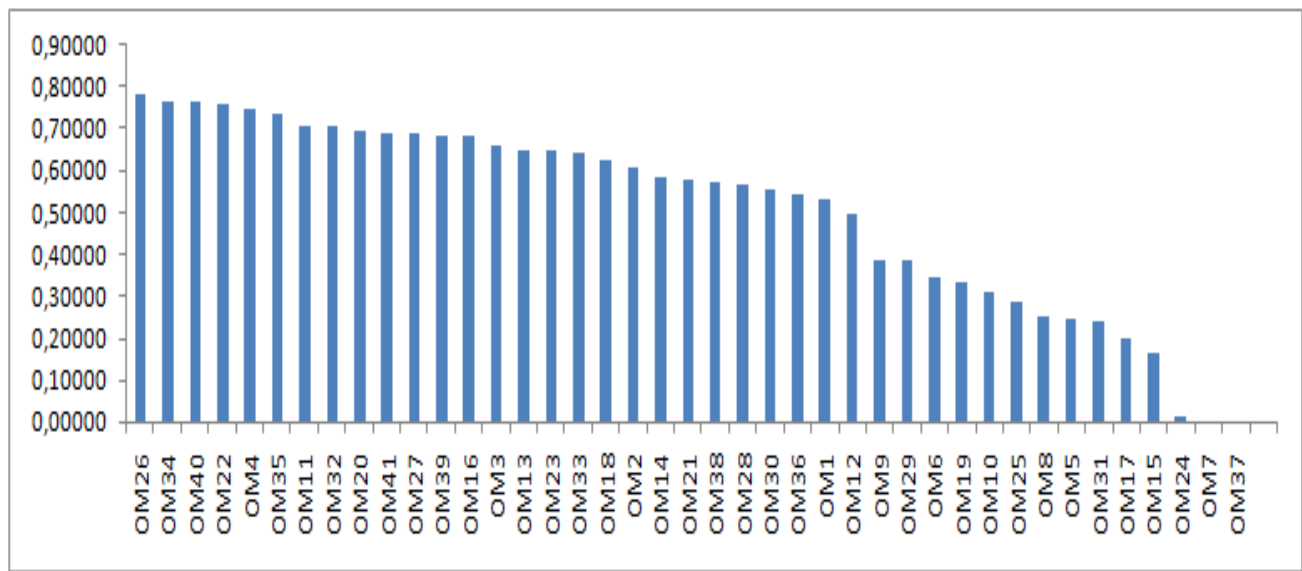

Fonte: Os autores com base em CHAGAS, 2019.

Observa-se, na Figura 8, que as cinco primeiras $\mathrm{OM}$ mais bem avaliadas na pesquisa são as OM26, OM34, OM40, OM22 e OM4.

\subsubsection{Resultados da Estimação do Modelo de Regressão Linear Múltipla (MRLM)}

As contribuições dos indicadores parciais (variáveis explicativas) para o indicador global podem ser quantificadas por meio de um MRLM, cujos resultados são apresentados na Tabela 3.

Tabela 3 - Importância relativa para cada tema nas 41 OM avaliadas 


\begin{tabular}{c|c|c|c|c}
\hline $\begin{array}{c}\text { Ambiente de } \\
\text { controle }\end{array}$ & $\begin{array}{c}\text { Avaliação de } \\
\text { riscos }\end{array}$ & $\begin{array}{c}\text { Atividades de } \\
\text { controle }\end{array}$ & $\begin{array}{c}\text { Informação e } \\
\text { comunicação }\end{array}$ & Monitoramento \\
\hline $15 \%$ & $28 \%$ & $18 \%$ & $8 \%$ & $31 \%$ \\
\hline
\end{tabular}

Fonte: CHAGAS, 2019.

Observa-se na Tabela 3 que o monitoramento é considerado importante para a harmonização das OM avaliadas. A componente avaliação de riscos atingiu o segundo lugar. A importância desses componentes contrasta com o estudo de Araújo (2014) sobre controles internos na Universidade Federal da Paraíba (UFPA). O autor destaca que componente monitoramento e avaliação de riscos apresentam o menor índice de alinhamento do sistema de controle interno da Universidade (CHAGAS, 2019).

\subsubsection{Resultados da Análise de Agrupamentos (AA)}

A aplicação da Análise de Agrupamentos (AA) aos valores dos indicadores globais visa formar grupo de OM semelhantes no que tange a harmonização com a abordagem COSO.

A seguir, a Tabela 4 apresenta a classificação das OM avaliadas em quatro clusters identificados pelas cores azul (muito alto), verde (alta), amarelo (moderada) e vermelho (baixa), conforme indicado na Figura 9.

Tabela 4 - Classificação das OM avaliadas

\begin{tabular}{c|r|c|r|c|c}
\hline OM & Indicador global & OM & Indicador global & Indicador global & \multicolumn{2}{c}{ OM } \\
\hline OM26 & 0,78538 & OM32 & 0,70543 & OM13 & 0,65055 \\
\hline OM34 & 0,76480 & OM20 & 0,69568 & OM23 & 0,64757 \\
\hline OM40 & 0,76301 & OM41 & 0,68826 & OM33 & 0,64087 \\
\hline OM22 & 0,76078 & OM27 & 0,68812 & OM18 & 0,62580 \\
\hline OM4 & 0,75040 & OM39 & 0,68666 & OM2 & 0,60799 \\
\hline OM35 & 0,73460 & OM16 & 0,68557 & & \\
\hline OM11 & 0,70686 & OM3 & 0,65915 & & \\
\hline OM 14 & 0,58601 & OM28 & 0,56499 & OM1 & 0,53349 \\
\hline OM21 & 0,57918 & OM30 & 0,55381 & OM12 & 0,49763 \\
\hline OM38 & 0,57206 & OM36 & 0,54346 & & \\
\hline OM9 & 0,38372 & OM10 & 0,31097 & OM31 & 0,24012 \\
\hline OM29 & 0,38327 & OM25 & 0,28739 & OM17 & 0,19942 \\
\hline OM6 & 0,34245 & OM8 & 0,24981 & OM15 & 0,16534 \\
\hline OM19 & 0,33158 & OM5 & 0,24730 & & \\
\hline OM24 & 0,01300 & OM7 & 0,00000 & OM37 & 0,00000 \\
\hline
\end{tabular}

Fonte: Adaptada de CHAGAS, 2019.

Figura 9 - Escala construída para os agrupamentos 


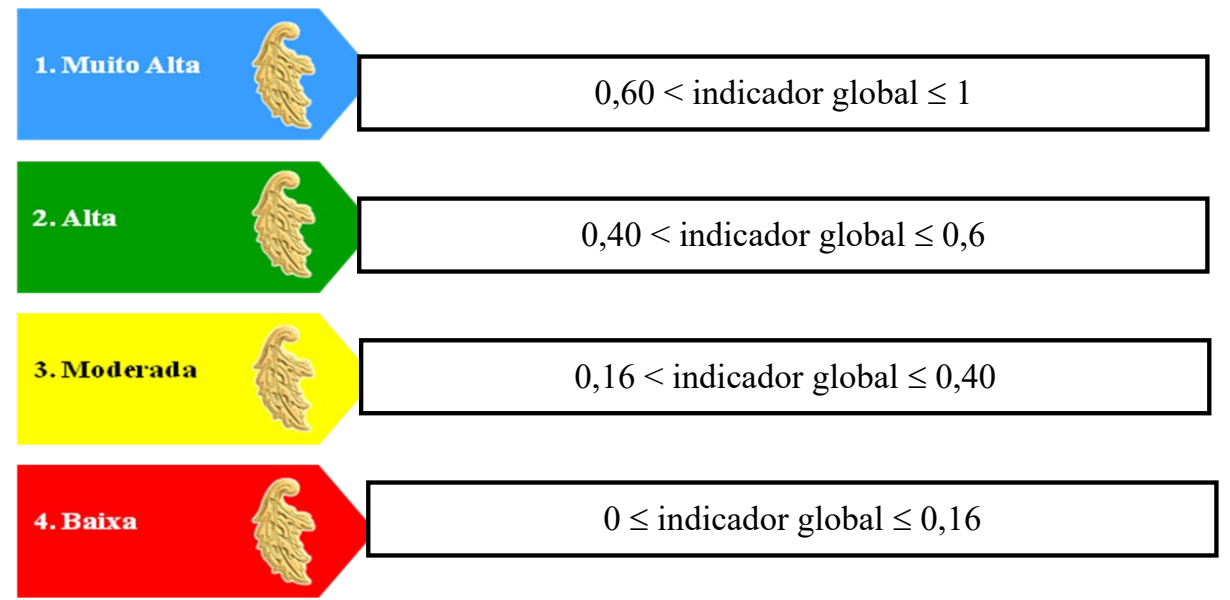

Fonte: CHAGAS, 2019.

Constata-se que o agrupamento 4 apresenta as OM com menor harmonização com a abordagem COSO. Já o agrupamento 1 relaciona as OM com maior harmonização.

A seguir, na Figura 10, busca-se identificar os pontos fortes e fracos de cada cluster, por meio de boxplots dos indicadores parciais em cada tema avaliado.

Figura 10 - Boxplots dos indicadores parciais
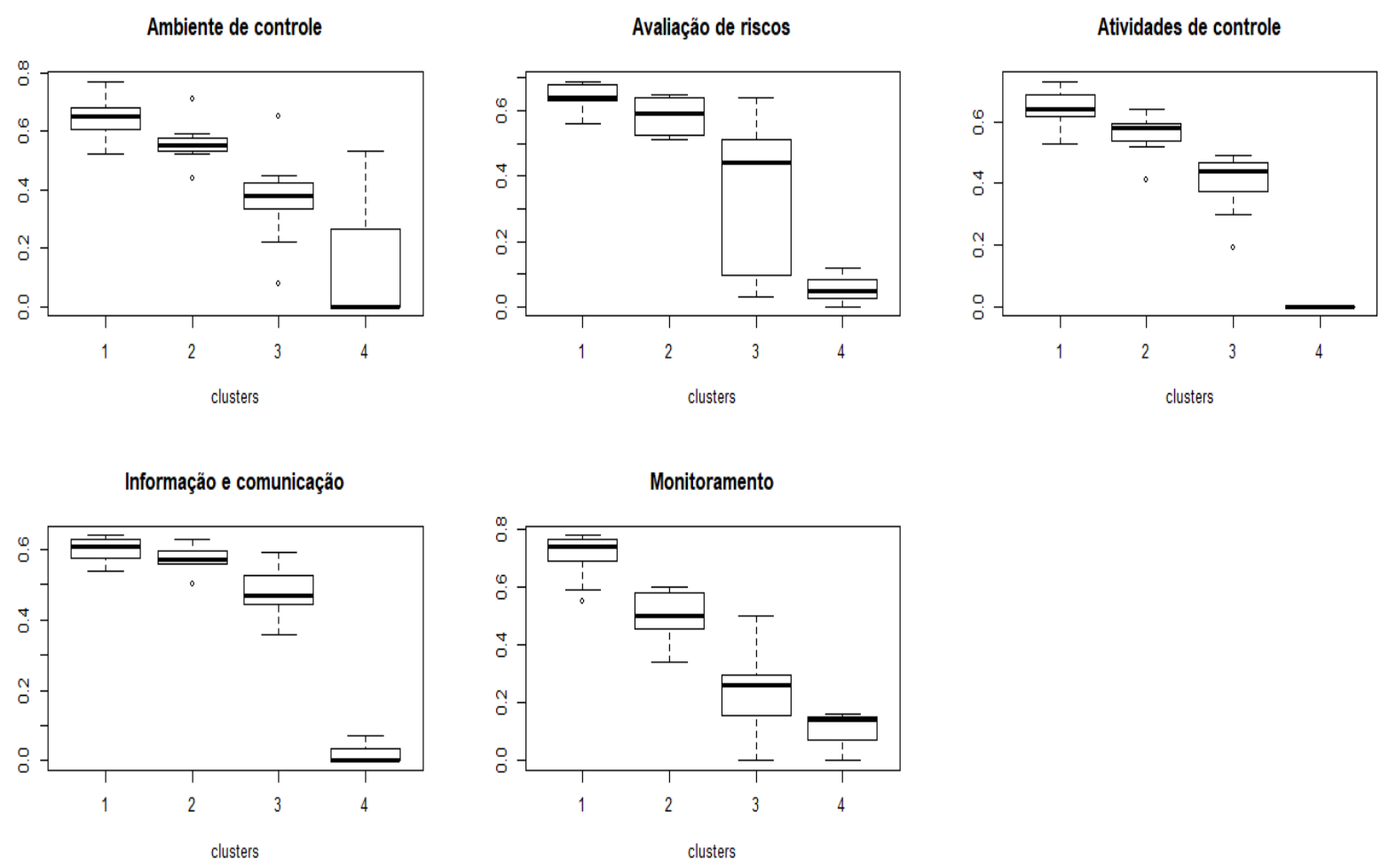

Fonte: CHAGAS, 2019.

Conforme ilustrado na Figura 10, as OM classificadas no cluster 1 possuem as maiores medianas em todos os temas, logo o cluster 1 representa o padrão de referência (benchmark) para os outros clusters no que tange a harmonização com a abordagem COSO.

Ainda na Figura 10, observa-se que no cluster 1 estão as $\mathrm{OM}$ sem pontos fracos, bem harmonizadas com o COSO. Já no cluster 2 estão as OM com pontos fortes em 2 temas 
(avaliação de riscos e informação e comunicação) enquanto que no cluster 3 algumas OM possuem pontos fortes em um determinado tema, por exemplo, ambiente de controle. O cluster 4 apresenta OM sem pontos fortes, com baixíssima harmonização com o COSO.

\section{CONSIDERAÇÕES FINAIS}

O presente estudo desenvolveu um diagnóstico da harmonização das estruturas de controle interno na Marinha do Brasil (MB) com a abordagem COSO.

Após uma revisão da literatura, aplicou-se um questionário estruturado submetido aos responsáveis pelo controle interno de 41 Organizações Militares (OM) da Marinha do Brasil (MB).

A análise dos dados foi realizada em duas partes. A primeira aborda os aspectos relacionados ao perfil dos respondentes. A segunda mostra os resultados encontrados na avaliação da harmonização das estruturas de controle interno na MB com a abordagem COSO por meio da aplicação de técnicas estatísticas multivariadas (Análise de Correspondência Múltipla (ACM), Análise de Componentes Principais (ACP), Modelo Regressão Linear Múltipla (MRLM) e Análise de Agrupamentos (AA)).

O estudo revelou que a maioria dos responsáveis pelo controle interno nas OM avaliadas são do sexo masculino (75,6\%), com formação acadêmica na área de administração (32\%). Os mesmos são do quadro de intendência (51,25\%) e oficiais superiores $(58,5 \%)$.

No que tange aos resultados descritos sobre a avaliação da harmonização das estruturas de controle interno na MB com a abordagem COSO, o estudo aponta que a maioria das OM avaliadas apresentou bons indicadores parciais nos temas, o que considera que há uma boa harmonização na estrutura de controle interno.

Adicionalmente, as contribuições dos componentes da abordagem COSO para o indicador global não são uniformes e apenas três componentes concentram quase $75 \%$ das contribuições. As atividades de monitoramento fornecem a maior contribuição para o indicador global da harmonização das estruturas de controle interno na MB com a abordagem COSO (31\%). Em segundo lugar aparece a avaliação de riscos (28\%), seguido do ambiente de controle (15\%).

No tocante a segmentação das OM avaliadas em agrupamentos semelhantes no que tange a harmonização com a abordagem COSO, os resultados revelam a classificação das OM em 4 agrupamentos. O estudo evidenciou que o agrupamento 4 está associado a menor harmonização, com três OM, enquanto que o agrupamento 1 apresenta as OM mais harmonizadas (19 OM).

Visando aprofundar novas descobertas sobre controle interno com abordagem COSO, sugere-se a ampliação da pesquisa em outras instituições do setor público no âmbito estadual, municipal e federal. Além disso, para estudos futuros, sugere-se que a Marinha do Brasil (MB) adote as mesmas técnicas estatísticas multivariadas utilizadas no presente estudo em outros temas relacionados à administração e contabilidade com objetivo de contribuir para a avaliação da gestão pública, proporcionando uma administração com mais transparência, eficiência e economicidade de recursos públicos.

\section{REFERÊNCIAS BIBLIOGRÁFICAS}

BRANDÃO, J. F. Sistemas de controle interno e transparência pública: estudo de caso da Universidade Federal de Alagoas. 2015. 135 p. Dissertação (Mestrado em Ciências Contábeis, Atuariais e Finanças) - Pontifícia Universidade Católica de São Paulo, São Paulo, 2015. 
BRASIL. Constituição (1988). Constituição da República Federativa do Brasil. Brasília, DF: Senado, 1988.

Decreto $\mathrm{n}^{0}$ 5.417, de 13 de abril de 2005. Aprova a Estrutura Regimental e o Quadro Demonstrativo dos Cargos em Comissão e das Funções Gratificadas do Comando da Marinha, do Ministério da Defesa, e dá outras providências. Diário Oficial [da] República Federativa do Brasil, Brasília, DF, 13 abr. 2005.

Lei $n^{\circ}$ 4.320, de 17 de março de 1964. Estatui Normas Gerais de Direito Financeiro para elaboração e controle dos orçamentos e balanços da União, dos Estados, dos Municípios e do Distrito Federal. Diário Oficial [da] República Federativa do Brasil, Brasília, DF, 23 mar. 1964.

. Lei Complementar $n^{0} 101$, de 04 de maio de 2000. Estabelece normas de finanças públicas voltadas para a responsabilidade na gestão fiscal e dá outras providências. Diário Oficial [da] República Federativa do Brasil, Brasília, DF, 04 mai. 2000.

BRITO, J. R. Breve Histórico do Controle Interno do Poder Executivo Federal: Origem, Evolução, Modelo Atual e Visão de Futuro. Revista de Negócios, n. 7, p. 1-24, 2009.

CANNAVINA, V. C. Os controles internos das entidades da administração pública direta do Poder Executivo Federal: um estudo baseado nos relatórios de gestão referentes à prestação de contas anual ao Tribunal de Contas da União. 2017. 190 f. Dissertação (Mestrado em Ciências Contábeis) - Fundação Escola de Comércio Álvares Penteado FECAP, São Paulo, 2014.

CARDIN, S. A.; GUIMARÃES, F. R. F. B.; BARBOSA, M. V.; MONTEIRO, A. S.; LOPES, P. L. Controle Interno na Administração Pública como Ferramenta para Economicidade de Processos. In: SIMPÓSIO DE EXCELÊNCIA EM GESTÃO E TECNOLOGIA, 12. 2015. Resende/RJ.

CARVALHO, H. Anállise Multivariada de Dados Qualitativos: Utilização da Análise de Correspondências Múltiplas com o SPSS. Edições Silabo. Lisboa, 2008.

CASTRO, D. P. Auditoria, Contabilidade e Controle Interno no Setor Público. 6. ed. São Paulo: Atlas. 2015.

CONTI, J. M.; CARVALHO, A. C. O Controle Interno na Administração Pública Brasileira: Qualidade do Gasto Público e Responsabilidade Fiscal. Revista de Direito Público, v. 8, n.37, p. 1-20, 2011.

COMMITTEE OF SPONSORING ORGANIZATIONS OF THE TREADWAY COMMISSION (COSO). Internal Control - Integrated Framework, 1994.

. Controle Interno - Estrutura Integrada. Tradução de: Instituto dos Auditores Internos do Brasil (IIA) e PricewaterhouseCoopers (PWC). 2013.

Gerenciamento de Riscos Corporativos - Integrado com Estratégia e Performance - Sumário Executivo. Tradução de: Instituto dos Auditores Internos do Brasil (IIA) e PricewaterhouseCoopers (PWC). 2017.

CHAGAS, C. A. S. Diagnóstico da harmonização das estruturas de controle interno na Marinha do Brasil com a abordagem COSO. 2019. 160 f. Dissertação (Mestrado em Ciências Contábeis) - Faculdade de Administração e Finanças, Universidade do Estado do Rio de Janeiro, Rio de Janeiro, 2019.

CREPALDI, S. A. Contabilidade Gerencial: Teoria de Prática. 7. ed. São Paulo: atlas, 2012. 
GATTRINGER, J. L. O controle interno na administração pública: Um estudo nos municípios catarinenses. 2016. 170 f. Dissertação (Mestrado em Administração) Universidade do Vale do Itajaí, Biguaçu - SC, 2016.

MALHOTRA, N. K. Pesquisa de marketing: uma orientação aplicada. 6. ed. Porto Alegre: Bookman, 2012.

MENEZES, S. M. M.; LIBONATI, J. J.; NEVES, T. J. G. Funcionamento dos Controles Internos e o Modelo Utilizado pelo Committee of Sponsoring Organizations of the Treadway Commission (COSO): Um Estudo da Percepção dos Gestores da Universidade Federal de Pernambuco. In: CONGRESSO USP DE CONTROLADORIA E CONTABILIDADE, 15, 2015. São Paulo. São Paulo. Anais... Disponível em: <http://www.congressousp.fipecafi.org/ anais/artigos152015/230.pdf>. Acesso em: 02 abr 2019.

MINISTÉRIO DA TRANSPARÊNCIA E CONTROLADORIA-GERAL DA UNIÃO-CGU. Instrução Normativa $n^{0}$ 3, de 09 de junho de 2017. Aprova o Referencial Técnico da Atividade de Auditoria Interna Governamental do Poder Executivo Federal.

MINGOTI, S. A. Análise de dados através de métodos de estatística multivariada - Uma abordagem aplicada. 1. ed. 3. reimpressão. Minas Gerais: UFMG. 2017.

NEVES, L. T.; PESSANHA, J. F. M.; SANTOS, N. M. G. Uso combinado de técnicas estatísticas multivariadas no desenvolvimento de indicadores de satisfação dos clientes de uma rede hospital. Revista Pesquisa Naval, Brasília, n. 25, p. 12-25, 2013.

PORTO, A. R. F. Avaliação da eficácia do sistema de controle interno do comando da aeronáutica brasileira. Portugal, 2012. 129 f. Dissertação (Mestrado em Administração Pública) - Universidade do Minho, Portugal, 2012.

SARTORIO, S. D. Aplicações de técnicas de análise multivariada em experimentos agropecuários usando o software R. 2008. 130 f. Dissertação (Mestrado em Agronomia) Universidade de São Paulo, Piracicaba, 2008.

SILVA, D. R.; BONACIM, C. A. G. A influência da lei de responsabilidade fiscal na gestão das contas públicas segundo a percepção de um contador. Sociedade, Contabilidade $e$ Gestão, Rio de Janeiro, v. 5, n. 2, p. 148-168, 2010.

TEIXEIRA, E. C. B. Estudo dos controles internos das instituições de ensino superior públicas do estado do Paraná. 2008. 193 p. Dissertação (Mestrado em Ciências Contábeis e Atuariais) - Pontifícia Universidade Católica de São Paulo, São Paulo. 